\title{
The Art of Counting - Interview with Mireille Bousquet-Mélou
}

Juanjo Rué (Universitat Politècnica de Catalunya, Spain, and Barcelona Graduate School of Mathematics, Spain)

Mireille Bousquet-Mélou received the Distinguished Speaker Award in 2017 from the European Mathematical Society. She delivered her talk entitled 'Functional equations in enumerative combinatorics' at the Foundations of Computational Mathematics conference in Barcelona, Spain. This interview took place in one of the cloisters at Universitat de Barcelona on 15 July 2017.

Dear Mireille, welcome to Barcelona. We are grateful that you could come to the 'Foundations of Computational Mathematics' conference in order to talk about combinatorics. We would like to ask you some questions, starting with your mathematical career path. Where do you come from? And as a child, did you have any mathematical reference in your family?

I was born in 1967 in Albi, a town in the south-west of France, not too far from Toulouse, but I grew up in Pau, where we moved with my family when I was three years old. My parents were both high school teachers, teaching history and geography, and there was no mathematician in the family. I have an elder sister too, who is also a teacher, in design and fine arts. Our tastes were different and that was good because each of us had our own space in the family. Who knows, maybe if she had liked mathematics, I would be good at drawing.

\section{So, do you have memories about your first contact with mathematics?}

There are a couple of them: in France we have écoles maternelles rather than kindergartens, where children are assumed to learn things as in school and not only through play. I entered an école maternelle in 1970 when the Bourbaki group was having a huge influence on French society, in particular in school (an influence that the group had probably not sought). So, in école maternelle we learnt... set theory. I do not remember this in detail but my parents kept my notebook from my last year at école maternelle. There you can see pictures of a rabbit, a cat, a banana... and we had to build the set of animals and give its cardinality. So, my first encounter with mathematics was maybe with this baby version of set theory!

After this, in the first year at primary school, I remember that we learnt to count simultaneously in all bases, not just in base 10 (this was probably again due to Bourbaki's model in education). We used little cubes to count, arranged first by rows, then piled to form squares, which could then be piled into cubes...

What was the first moment you got interested in mathematics?

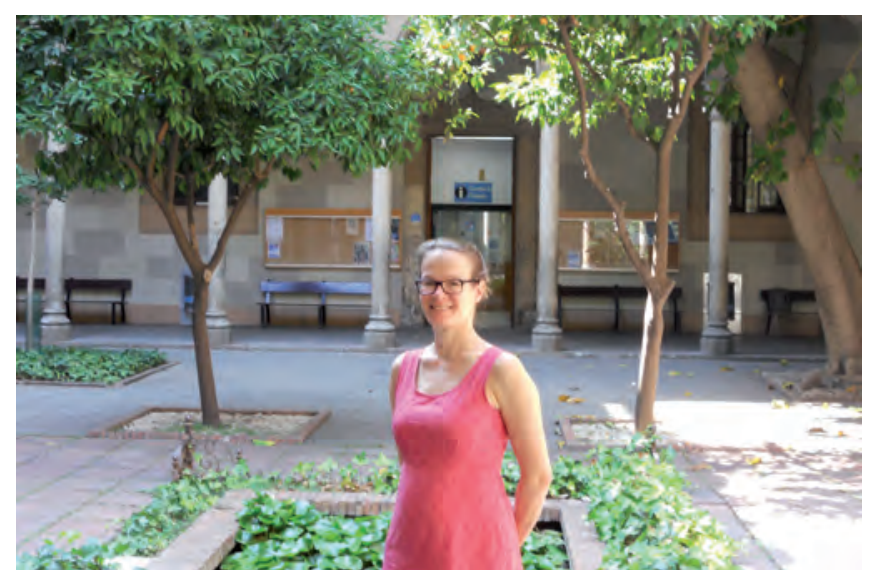

Mireille Bousquet-Mélou, Barcelona 2017. (Photo: Juanjo Rué)

It took some time: I was the type of good pupil at high school who was decently interested in many topics but not immensely fond of any. I think that my taste for mathematics grew in the last year of high school, where I had a rather peculiar course of mathematics. Our mathematics teacher did not lecture (I think it bored him) but he made us learn the material of the book at home. So the mathematics course at school was based on solving problems. However, the good pupils not only studied the book at home but also worked on the problems. This created a competitive environment where we learnt to work independently. I think this was very good for the good students and probably rather bad for the others. Anyway, I liked it: first working by myself and then discussing with the other pupils.

During high school, I did not have a clear plan of what I wanted to do next but, at that time in France, attending an engineering school sounded like a prestigious thing to do. The good students in my class wanted to become engineers so I used to say the same (even if I did not have any idea of what the job was like). Hence, after high school, I moved to a classe préparatoire ${ }^{1}$ in Toulouse where I studied mathematics and physics. There I learnt about the École Normale Supérieure (ENS). But I heard that students there would become professeurs. In France we use the same word (professeurs) for university professors and high school teachers. My parents were high school teachers, hence professeurs, and (of course) I wanted to do something different, so at the beginning I was not considering taking the entrance examination for the ENS.

French system parallel to university that provides training to enter the so-called grandes écoles, most of them being engineer schools. 
But you studied at the École Normale Supérieure anyway. How did you change your mind?

In the second year of my classes préparatoires, some former students who were studying at the ENS came to Toulouse to advertise what they were doing. They talked about research and the atmosphere they described seemed very exciting... and they even had a climbing club! This was clearly irresistible. So they convinced me to take the entrance examination and I was finally selected to go there.

You moved to Paris to start your studies at the ENS. How was the ENS at the time?

There were 45 new students entering the school in mathematics and I was the only woman. In fact, this was the first year that the ENS was a mixed school. The head of the school was of course not happy with this proportion (previously there had been 15 positions for women and 30 positions for men).

We could study mathematics of course but we could also move to computer science or physics (and we were somewhat encouraged to do so). But the professors there were keen to keep one woman in mathematics and I went on with mathematics. This was probably the best choice for me, since I realised later that physics is a different world, in which I think I would have felt less comfortable.

I enjoyed my time at the ENS a lot. There were a huge variety of people in (let us say) an educated atmosphere with diverse interests. Indeed, students specialised not only in science but also history, languages and sociology, among other topics. Even if you did not interact much with them, one could feel a different atmosphere compared to the one in the classes préparatoires or in engineer schools. Many students had (sometimes unusual) hobbies, ranging from contemporary music to spending nights in the Paris catacombs. It looked a bit as if everyone had to be special in some way (and had to show it). I did not feel such a need. Being a woman in mathematics was probably special enough.

\section{Who got you into research at the ENS? What were your favourite topics there?}

Research is the "normal" direction to take at the ENS. In the first year, we had to follow a number of courses and one of them was taught by Xavier Viennot on enumerative combinatorics. The type of mathematics he was presenting was very different from the material covered by the other professors. His style was very different too. He was teaching with plastic slides (not on the blackboard), putting six slides on top of each other and proving identities on functions by showing bijections between the objects that these functions counted. It was very attractive. I also took a couple of courses in probability theory by Marc Yor. They were not easy but I liked them a lot. I also remember a great course on complex analysis by Joseph Oesterle. I still use it!

In my second year, Xavier Viennot went abroad and I specialised with a master in probability theory and statistics. However, in the third year (when we were to choose a PhD topic), Xavier Viennot came back to France. He was living near Bordeaux but coming to Paris once a week to teach. I hesitated between probability and combinatorics. I had seen more of the first topic and only a small piece of combinatorics but this topic was still very tempting. So, in the end, it was combinatorics and I must say that I never had any regrets about this choice (even though I am delighted to be in touch and interacting with probabilists in my work).

After defending your PhD, you moved with a CNRS position to Bordeaux, at LaBRI (Laboratoire Bordelais de Recherche en Informatique). Can you describe the department when you moved to Bordeaux?

In France (and other places), combinatorics is at the border between mathematics and computer science. Laboratoire in French is similar to department, or institute, so LaBRI is, in fact, the Department of Computer Science at the University of Bordeaux. I was not there from the very beginning but I know that combinatorics played an important role in the early days of LaBRI, together with other aspects of theoretical computer science. The first combinatorialist there was Robert Cori; Xavier Viennot joined LaBRI a bit later. Both of them were former students of Marcel-Paul Schützenberger, who is the "father" (and grandfather and great-grandfather) of many combinatorialists in France. Robert Cori and Xavier Viennot were influential and attracted many people to combinatorics.

Of course, computer science has evolved very rapidly over the years: nowadays, our department combines a larger variety of topics, including parallel computation, image processing, bioinformatics and robotics. But we still have two strong groups in theoretical computer science.

Can you explain, in general terms, what your research area is and what its connection is with other areas of science?

I work in a field of discrete mathematics (or is it theoretical computer science...) called enumerative combinatorics. Most of the questions that we study start like this: given a set of discrete objects, equipped with a notion of size (say permutations on $n$ elements), how many objects of size $n$ are there? Of course you do not want a number for particular values of $n$ but a formula or, more realistically, a characterisation (e.g. a recurrence relation) valid for general $n$. The objects that we (try to) count come from various branches of mathematics, including probability (of course the interaction with this area is particularly strong via discrete probability), algebra (e.g. in connection with representations of classical groups and algebras) and mathematical physics (via the study of discrete models, like the famous Ising model).

Most French combinatorialists work in computer science departments. There are several reasons for that, partly historical but mostly scientific: there is no real boundary between some parts of theoretical computer science (e.g. the study of formal languages) and discrete mathematics. There is also a strong interaction between enumerative combinatorics and the study of the com- 
plexity of algorithms, as launched a long time ago by Don Knuth and pursued in France by Philippe Flajolet and his school. The rough idea is that in order to understand the complexity of an algorithm, one has to determine how many entries of a given length get processed in a given time - a well-posed bivariate counting problem.

Let me get back to the nature of enumerative combinatorics: sometimes, more important than getting a counting formula for a certain problem is the fact that to arrive at such a formula requires information about the combinatorial structure under study. Hence, counting is sometimes just a pretext and the important thing is to understand, or discover, a structure in some discrete objects. From this point of view, enumerative combinatorics does not differ much from other branches of mathematics.

How do you decide which combinatorial objects you want to study and which problems you choose to investigate?

This is a difficult question and, to my shame, I must say that for many years I did not really think about it. Some topics would come along that I liked and that was it. Now, I try to be (sometimes) more selective: there are the problems that you think that you'll be able to solve and, as you get older, you see more of them. But if you do not go further you will not surprise yourself. Surprising oneself by solving something more challenging is more fun... but also more risky. A certain balance is required.

\section{Can you explain something about your particular fa- vourite combinatorial objects?}

I like them all... Maybe one of my early favourites was the so-called lecture hall partitions. ${ }^{2}$ In the 1990s, a young Swedish colleague, Kimmo Eriksson, introduced them to me and we discovered surprising identities that we published in three papers. Then I did not work on this topic anymore but it has had ramifications and is still active. Recently, I saw a paper on them that did not even cite the papers with Kimmo: isn't this the sure sign that 'your' objects have grown up?

I like very much planar maps, which have recently become a popular topic in probability theory too. Another of my favourites (and not only mine!) is self-avoiding walks and our joint results with Nick Beaton, Jan de Gier, Hugo Duminil-Copin and Tony Guttmann. As you know, in 2010, there was this groundbreaking work of Duminil-Copin and Smirnov about the growth constant of self-avoiding walks in the honeycomb lattice, which solved a conjecture from Nienhuis stating that this value was $\sqrt{2+\sqrt{2}}$. This was a simple, short and inventive proof. Many people from our community spent time trying to figure out what results could be proved with these new ideas. Tony Guttmann suggested looking at a conjecture dealing with walks in a half plane interacting with the boundary. Finally, after some difficulties, we proved it. I was very glad to be involved in that work.

\footnotetext{
2 A sequence of $\mathrm{n}$ integers $\left(\lambda_{1}, \lambda_{2}, \ldots, \lambda_{n}\right)$ is a lecture hall partition if $0 \leq \lambda_{1} \leq \lambda_{2} / 2 \leq \ldots \leq \lambda_{n} / \mathrm{n}$.
}

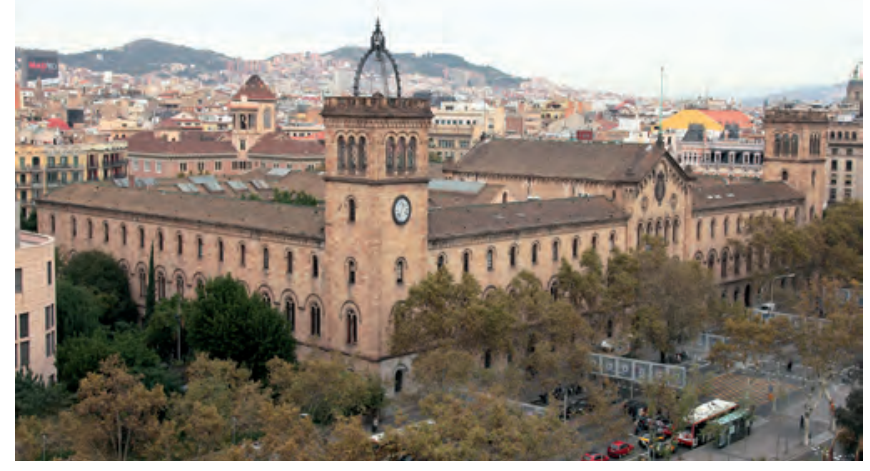

Historical building of Universitat de Barcelona, where FOCM took place. (Image courtesy of Universitat de Barcelona)

\section{Among your results, which one do you thing is your fa- vourite discovery?}

You can like your results for different reasons. For instance, I enjoyed this result about self-avoiding walks because I was doing something a bit different from my usual business, and of course because it is a difficult topic (though the questions are simple to state). I also like my early results about lecture hall partitions. Another topic that I am happy with is the enumeration of lattice walks confined in a cone. With Marni Mishna, we were maybe the first combinatorialists to consider the problem in a unified way, aiming at a complete classification. In the last 10 years, this topic has attracted people from different areas, ranging from computer algebra to differential Galois theory, via, of course, probability theory. In fact, several of these colleagues are here at FOCM. It is always fun when people away from your area get interested in 'your' questions.

\section{What do you find most rewarding in mathematical re- search?}

This is, of course, a matter of taste. There are some good things that you can rely on - for instance, I like giving talks. In general, I like writing papers and especially the time spent thinking about how to expose things. In the research itself, what I really like are those very few moments when you start feeling that you are about to understand something (sometimes without even knowing what this something is). You can feel that your ideas are evolving, that your brain is the right place for some alchemy to happen. This has happened to me a few times (at my modest scale) and it is definitely a remarkable feeling.

Of course, once you arrive at a certain result, you very often think: 'How is it that I did not realise this before; this is so simple!' But you still feel happy about having understood something, at last.

You were an invited speaker at ICM06 (Madrid) in the combinatorics section. Can you explain to us a little about this experience?

My first surprise was to be invited there. Then, being there was really good for me. I had never seen such a big crowd of mathematicians... This was very stimulating. The strange thing was that, being mostly by myself in 
this big crowd, I did not feel isolated: I was moving from plenary lectures to sessions, in probability and combinatorics. I realised that many plenary lectures were very good and understandable. This was when Wendelin Werner and Andreï Okounkov got their Fields Medals. There were presentations of their work, with a strong discrete flavour. There was also a plenary lecture by Richard Stanley, a specialist of enumerative and algebraic combinatorics.

I remember that at some point I was so enthusiastic that I started a long list of topics that I would like to look at. This list is still on my desk, though a bit faded. I have ticked as "done" some of its items but many are left! More than 10 years later, I still have a look at it every now and then. To be frank, a second newer list is now stapled to it.

Going back to the fact that you were the only woman in your year at the ENS: can you describe how your career path evolved related to the fact that you are a female mathematician?

Frankly, I did not feel real obstacles due to being a woman. But this does not mean that there are none. What I mean is that if I had had serious difficulties, I would maybe have received less recognition and you would not be interviewing me at the moment. By definition, the women who get exposure are among the happy few...

What strikes me the most in the women/science question is what our societies teach to teenagers: from many sources they hear that science (and especially mathematics) is not for women. I may be pessimistic, and exaggerating a bit, but my impression is that advertisements, movies, etc., still spread the idea that women should take care about their clothes and make-up and that being smart is not a priority. In a sense, it is unfortunate that young people start choosing what they want to study at an age when it is soooooo important to obey gender rules.

Even in our community, there is still this idea that a mathematician is a male mathematician. I would recommend trying the Implicit Association Tests developed by Harvard University: ${ }^{3}$ they are cleverly designed and you cannot really cheat them. In my case, I must say that they showed that I strongly correlate science and male gender... I do not know what it means nor whether it implies that my judgment is biased but the correlation is definitely there.

Fortunately, times are slowly changing: 15 years ago this problem was not as explicit as it is today. We are definitely more aware about these biases and we are trying to fight them.

What would you recommend to a young woman in Europe wanting to start and develop a career in mathematics?

Hold tight and do what you like! Times seem to be more favourable than before. For young women who are already inside academia, there is at least an effort in the mathematical community to fight bias. And to attract

3 https://implicit.harvard.edu/implicit/education.html.

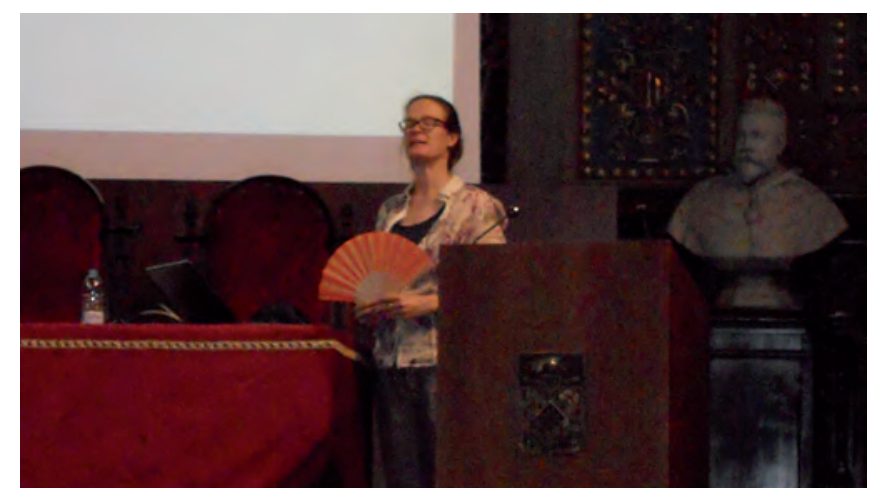

Mireille Bousquet-Mélou during her talk at FOCM. July in Barcelona is quite hot and fans provided by the organisation were greatly appreciated by all conference attendees. (Photo: Juanjo Rué)

more women, female scientists must probably get to talk more to young students at high school, as well as appearing on television and on the internet...

I am not an expert on these questions but I know that many universities explore how to help at various moments of a woman's career, like after maternity leave (e.g. less teaching when returning and grants to invite collaborators). And of course we should all have the representation of women in mind when organising a conference or gathering together a committee.

Just to finish, could you tell us the directions that should be followed by enumerative combinatorics in the near future?

This is a difficult question. I would say that problems interacting with other areas (of mathematics, or even further) will play an important role in combinatorics. But maybe it would be more honest to say that this is the type of combinatorics that I like.

Here at FOCM, I attend talks at the combinatorics workshop of course but also the computer algebra workshop (ah, no; they say 'symbolic analysis' so it must be a bit different). I am by no means an expert but I use some computer algebraic tools, for instance to handle classes of formal power series (generating functions, in our combinatorial language). There are also some colleagues here from other areas of mathematics, like number theory, who ask different questions about the same classes of series. I like this mixture. I like to hear about these other perspectives, even if it is likely that I will never work with these colleagues. Well, after all, who knows?

\section{Thank you very much Mireille.}

Thank you

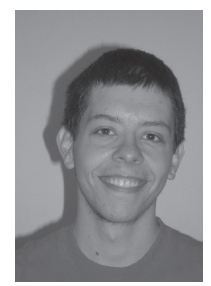

Juanjo Rue is an associate professor at the Department of Mathematics of Universitat Politècnica de Catalunya and a faculty member at the Barcelona Graduate School of Mathematics. His research interests are enumerative combinatorics, random graphs, additive combinatorics and extremal combinatorics. 